\title{
Interaction of oxygen vacancies with domain walls and its impact on fatigue in ferroelectric thin films
}

\author{
Yu Xiao and Kaushik Bhattacharya \\ Division of Eng. and Appl. Sci., California Institute of Technology, Pasadena, CA 91125, USA
}

\begin{abstract}
The role of oxygen vacancies in fatigue and dielectric breakdown has been a topic of intense research in ferroelectric perovskites like $\mathrm{BaTiO}_{3}$. This paper presents a comprehensive model that treats the ferroelectrics as polarizable wide band-gap semiconductors where the oxygen vacancies act as donors. First, a fully coupled nonlinear model is developed with space charges, polarization, electric potential and elastic displacements as variables without making any a priori assumptions on the space charge distribution and the polarization. Second, a Pt/BaTiO $3 / \mathrm{Pt}$ structure is considered. Full-field coupled numerical simulations are used to investigate the structure of $180^{\circ}$ and $90^{\circ}$ domain walls in both perfect and defected crystals. The interactions of oxygen vacancies with domain walls are explored. Numerical results show that there is pronounced charge trapping near $90^{\circ}$ domain walls, giving rise to possible domain wall pinning and dielectric breakdown. Third, a simple analytical solution of the potential profile for a metal/ferroelectric semiconductor interface is obtained and the depletion layer width is estimated. These analytical estimates agree with our numerical results and provide a useful tool to discuss the implications of our results.
\end{abstract}

Keywords: Ferroelectric thin film, wide band-gap semiconductor, oxygen vacancy, fatigue, domain wall

\section{INTRODUCTION}

Fatigue or the loss of switchable polarization under bipolar cycling and dielectric breakdown are two critical issues which have hindered the commercialization of ferroelectric memory devices. Therefore they have received considerable attention in recent years. Various mechanisms have been postulated based on substantial experimental data, and most of them are interwoven in one way or the other. ${ }^{1-4}$ Although none of the mechanisms is unanimously accepted, it is widely recognized that under most circumstances injected charge trapping and oxygen vacancy trapping/accumulation are very likely responsible for ferroelectric fatigue. ${ }^{5-8}$ However, these two mechanism are yet to be fully developed since the very reasons for their occurrence and their relations are still under debate. Most explanations are essentially phenomenological, and a systematic and quantitative model is still missing. The present paper building on the preliminary ideas of Shenoy and Bhattacharya ${ }^{9}$ is an effort along this direction.

We start with ferroelectric perovskites with oxygen vacancies. The idea that oxygen vacancies may play an important role in ferroelectric fatigue and dielectric breakdown is based on several experimental observations:

- $\mathrm{YBCO} / \mathrm{PZT} / \mathrm{YBCO},{ }^{10} \mathrm{SRO} / \mathrm{PZT} / \mathrm{SRO}^{8}$ and $\mathrm{Pt} / \mathrm{SBT} / \mathrm{Pt}^{11}$ are almost fatigue-free compared to the pronounced fatigue in $\mathrm{Pt} / \mathrm{PZT} / \mathrm{Pt}$;

- Oxygen vacancies are redistributed during polarization fatigue, ${ }^{12,13}$ higher oxygen vacancy concentration near electrodes are observed;

- Oxygen-poor annealing can also cause suppression of switchable polarization, and this suppression can not be restored by UV/bias combination but can be restored by an oxygen-rich re-annealing. ${ }^{6}$

Author of correspondence: K. Bhattacharya.

E-mail: bhatta@caltech.edu.

Tel: 1-626-395-8306; fax: 1-626-568-2719. 
In addition, oxygen vacancies are the most mobile ionic defects in perovskite material, and thus the most feasible candidates for fatigue caused by defect chemistry.

In this paper, we develop a model that treats the perovskite ferroelectrics as a diffusible, deformable, polarizable semiconducting solids. It is well known that perovskite ferroelectrics are wide band-gap semiconductors ${ }^{14}$ : the band-gap for $\mathrm{BaTiO}_{3}$ is $3.0 \mathrm{eV}$, and $\mathrm{PbZr}_{0.40} \mathrm{Ti}_{0.60} \mathrm{O}_{3}$ is $3.4 \mathrm{eV}$. Further, oxygen vacancies act as donors or n-type dopants for the ferroelectric. This model which combines the ferroelectric and semiconducting aspects of perovskites allows us to investigate the interaction of oxygen vacancies with domain walls. In particular, it allows us to study the space charge redistribution in the ferroelectric in the presence of electrodes and domain walls. We examine a Pt/ $\mathrm{BaTiO}_{3} / \mathrm{Pt}$ capacitor in detail, and find the formation of depletion layers at the ferroelectricelectrode boundary as electrons diffuse from the $\mathrm{BaTiO}_{3}$ to the $\mathrm{Pt}$ and also a trapped layer of electrons at $90^{\circ}$ domain walls. These are accompanied by significant electric field and in turn leads to field-driven diffusion of oxygen vacancies. All of this has important implications for dielectric breakdown and fatigue through domain wall pinning. Finally, our results also show significant stress/strain concentrations near the domain walls and at the intersections of domain walls with electrodes. These stress/strain concentration sites may likely serve as starting points for microcracking or domain wall pinning.

The paper is organized as follows. In Section 2, we derive our governing equations without making any a priori assumptions on the space charge distribution and the polarization. We obtain a set of fully coupled nonlinear equations with space charges, polarization, electric potential and elastic displacements as variables. In this derivation, we follow standard methods of continuum mechanics, calculating the dissipation and looking for dual pairings.

We consider perfect crystals in Section 3. Specifically, we consider a $\mathrm{Pt} / \mathrm{BaTiO}_{3} / \mathrm{Pt}$ capacitor with shorted electrodes. A Landau-Ginzburg-Devonshire type energy is chosen to describe the stored energy for $\mathrm{BaTiO}_{3}$. We obtain the detailed structures of $180^{\circ}$ and $90^{\circ}$ domain walls in two dimensions using finite element analysis. In addition, the stress/stain and electric field around the domain walls are also obtained and their implication on ferroelectric fatigue is discussed.

In Section 4, we also consider $\mathrm{Pt} / \mathrm{BaTiO}_{3} / \mathrm{Pt}$ capacitors, but with n-type doped $\mathrm{BaTiO}_{3}$. We investigate the interaction of oxygen vacancies with domain walls using finite element analysis. We also analytically analyze the band structure across the metal/ferroelectric semiconductor interface and obtain a simple expression for depletion layer width. We compare our computational results with analytic results; We finally discuss the impact of oxygen vacancies and domain walls on ferroelectric fatigue.

We conclude in Section 5 with a discussion.

\section{A CONTINUUM MODEL}

Consider a ferroelectric semiconducting crystal in an external field shown in Fig 1. The ferroelectric semiconducting crystal occupies a region $\Omega \subset \mathbb{R}^{3}$ in reference configuration and undergoes a deformation $\mathbf{y}: \Omega \rightarrow \mathbb{R}^{3}$. $\mathbf{y}(\Omega) \subset \mathbb{R}^{3}$ is the region it occupies in current configuration. $C_{v}$ are electrodes with fixed potential, $C_{q}$ are electrodes with fixed charges. The deformation gradient is defined as $\mathbf{F}=\nabla_{\mathbf{x}} \mathbf{y}$, and we assume $J=\operatorname{det} \mathbf{F}>0$ almost everywhere in $\Omega$.

We denote by $\mathbf{p}: \mathbf{y}(\Omega) \rightarrow \mathbb{R}^{3}$ the polarization of ferroelectric material per unit current volume, and by $\mathbf{p}_{0}: \Omega \rightarrow \mathbb{R}^{3}$ the polarization per unit reference volume,

$$
\mathbf{p}_{0}(\mathbf{x})=\left(\operatorname{det} \nabla_{\mathbf{x}} \mathbf{y}(\mathbf{x})\right) \mathbf{p}(\mathbf{y}(\mathbf{x}))
$$

The total charge density at any point in a semiconductor in current configuration is:

$$
\rho=e\left(z N_{d}-z^{\prime} N_{a}-n_{d}-n_{c}+p_{a}+p_{v}\right)
$$

where $N_{d}$ is the density of donors, $N_{a}$ the density of acceptors; $n_{d}$ is the density of electrons in donor's level, $n_{c}$ the density of electrons in conduction band; $p_{a}$ is the density of holes in acceptor's level, $p_{v}$ the density of holes in vacancy band; $z$ and $z^{\prime}$ are the valency of donors and acceptors respectively, and $e$ is the coulomb charge per electron. 


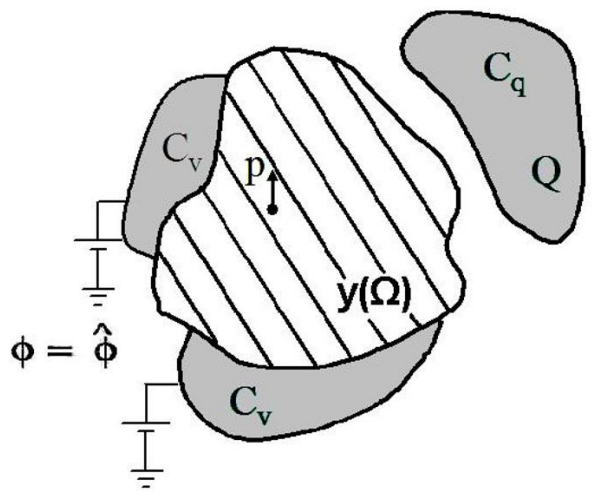

Figure 1. A ferroelectric semiconducting system in an external field generated by conductor $C_{q}$ and $C_{v}$.

We assume that oxygen vacancies are the dominant impurities in the semiconductor being considered. Since oxygen vacancies act as donors, we may set $N_{a}=p_{a}=0$ in Eq. 2. So,

$$
\rho=e\left(z N_{d}-n_{d}-n_{c}+p_{v}\right)=e\left(z N_{d}-n_{d}\right)+e\left(p_{v}-n_{c}\right)=e z N_{d}+\rho_{c}
$$

with

$$
\rho_{c}=e\left(-n_{d}+p_{v}-n_{c}\right) .
$$

We define the counterparts of $N_{d}, \rho, \rho_{c}$ in reference configuration as $N_{d 0}, \rho_{0}, \rho_{c 0}$ respectively. Assuming that no oxygen vacancies or charges are generated in the interior, we have the following continuity equations:

$$
\begin{gathered}
\dot{N}_{d 0}=-\nabla_{\mathbf{x}} \cdot \mathbf{J}_{N_{d 0}} \\
\dot{\rho}_{0}=-\nabla_{\mathbf{x}} \cdot \mathbf{J}_{\rho_{0}}=-\nabla_{\mathbf{x}} \cdot\left(e z \mathbf{J}_{N_{d 0}}+\mathbf{J}_{\rho_{c}}\right) .
\end{gathered}
$$

The total energy of the system consists two parts: the energy stored in ferroelectric material and the electric field energy generated by external and internal sources, i.e.

$$
\mathcal{E}=\int_{\Omega} W_{0} d x+\frac{\epsilon_{0}}{2} \int_{\mathbb{R}^{3}}|\nabla \phi|^{2} d x .
$$

Here, $W_{0}$ is the stored energy per unit reference volume in ferroelectric material, it depends on defect density $N_{d 0}$, total charge density $\rho_{0}$, polarization $\mathbf{p}_{0}$, polarization gradient $\nabla_{\mathbf{x}} \mathbf{p}_{0}$, and deformation gradient $\nabla_{\mathbf{x}} \mathbf{y}$, i.e., $W_{0}=W_{0}\left(N_{d 0}, \rho_{0}, \mathbf{p}_{0}, \nabla_{\mathbf{x}} \mathbf{p}_{0}, \nabla_{\mathbf{x}} \mathbf{y}\right)$. The electrical potential $\phi$ is obtained by solving Maxwell equation:

$$
\nabla \cdot\left(-\epsilon_{0} \nabla \phi+\mathbf{p} \chi(y(\Omega))\right)=\rho \quad \text { in } \mathbb{R}^{3}
$$

with boundary conditions:

$$
\begin{gathered}
\int_{\partial C_{q}} \frac{\partial \phi}{\partial \mathbf{n}} d \mathbf{a}=-\frac{Q}{\epsilon_{0}}, \quad \nabla \phi=0 \quad \text { in } C_{q}, \\
\phi=\hat{\phi} \quad \text { in } C_{v}, \\
\phi \rightarrow 0 \quad \text { as }|x| \rightarrow \infty
\end{gathered}
$$

The dissipation of the whole system $\mathcal{D}$ is defined as

$$
\mathcal{D}=\mathcal{F}-\frac{d \mathcal{E}}{d t}
$$


where $\mathcal{F}$ is the work done by external sources. This includes the mechanical work done by external forces, the electric work done by electrodes, and the chemical energy flux from $C_{v}$ into $\Omega$,

$$
\mathcal{F}=\hat{\phi} \int_{\partial \mathbf{y}\left(C_{v}\right)} \sigma d \mathbf{S}_{\mathbf{y}}+\int_{\partial \mathbf{y}\left(\Omega_{s}\right)} \mathbf{t} \cdot \dot{\mathbf{y}} d \mathbf{S}_{\mathbf{y}}-\int_{\partial \Omega} \mu_{N_{d 0}} \mathbf{J}_{N_{d 0}} \cdot \hat{\mathbf{m}} d \mathbf{S}_{\mathbf{x}}-\int_{\partial \Omega} \mu_{\rho_{c 0}} \mathbf{J}_{\rho_{c 0}} \cdot \hat{\mathbf{m}} d \mathbf{S}_{\mathbf{x}}
$$

where $\mu_{N_{d 0}}$ and $\mu_{\rho_{c 0}}$ are respectively the chemical potential carried by $N_{d 0}$ and $\rho_{c 0}$ flux; $\partial \mathbf{y}\left(\Omega_{s}\right)$ is the part of $\partial \mathbf{y}(\Omega)$ where external traction exists; $d \mathbf{S}_{\mathbf{y}}$ and $d \mathbf{S}_{\mathbf{x}}$ are differential area in current and reference configuration respectively; $\hat{\mathbf{m}}$ is the normal to surface in reference configuration and $\hat{\mathbf{n}}$ its counterpart in current configuration.

Substituting Eq. 7, 13 into Eq. 12, we go through a series of manipulations ${ }^{15}$ using Eq. 5, 6, 8 to write the dissipation as the sum of multiples of conjugated pairs (generalized velocities times generalized forces). This allows us to identify the governing equations following standard methodology in continuum mechanics (arguments following Coleman-Noll ${ }^{16}$ ):

$$
\begin{aligned}
& \nabla_{\mathbf{x}}\left(\frac{\partial W_{0}}{\partial \nabla_{\mathbf{x}} \mathbf{p}_{0}}\right)-\frac{\partial W}{\partial \mathbf{p}_{0}}-\mathbf{F}^{-T} \nabla_{\mathbf{x}} \phi=\mu \dot{\mathbf{p}}_{0} \quad \text { in } \quad \Omega, \\
& \frac{\partial W_{0}}{\partial \nabla_{\mathbf{x}} \mathbf{p}_{0}} \hat{\mathbf{m}}=0 \quad \text { on } \quad \partial \Omega, \\
& \left(\boldsymbol{\sigma}_{i j}+\mathbf{T}_{M i j}\right)_{, j}=0 \quad \text { in } \mathbf{y}(\Omega), \\
& \boldsymbol{\sigma} \hat{\mathbf{n}}-\llbracket \mathbf{T}_{M} \hat{\mathbf{n}} \rrbracket-\mathbf{t} \chi\left(\partial \mathbf{y}\left(\Omega_{s}\right)\right)=0 \quad \text { on } \quad \partial \mathbf{y}(\Omega),
\end{aligned}
$$

and

$$
\begin{aligned}
\frac{\partial W_{0}}{\partial N_{d 0}}-\mu_{N_{d 0}}+e z \phi+e z \mu_{\rho_{c 0}} & =0 & & \text { in } \Omega, \\
\frac{\partial W_{0}}{\partial \rho_{0}}+\phi-\mu_{\rho_{c 0}} & =0 & & \text { in } \Omega, \\
\mathbf{J}_{N_{d 0}} & =-k_{1} \nabla_{\mathbf{x}} \mu_{N_{d 0}} & & \text { in } \Omega, \\
\mathbf{J}_{\rho_{c 0}} & =-k_{2} \nabla_{\mathbf{x}} \mu_{\rho_{c 0}} & & \text { in } \Omega,
\end{aligned}
$$

where $\mu, k_{1}$ and $k_{2}$ are some constants great or equal to zero.

The first two equations, Eq. 14 and 15 are, respectively, the equilibrium equation of polarization and its boundary condition. Eq. 16 is the force equilibrium equation with boundary condition 17 . Notice that in the force equilibrium equation 16, we introduced two stress tensors, Cauchy stress tensor $\boldsymbol{\sigma}$ and Maxwell stress tensor $\mathbf{T}_{M}$, which are defined as

$$
\begin{aligned}
\boldsymbol{\sigma} & =\frac{1}{J}\left(\frac{\partial W_{0}}{\partial \mathbf{F}}\right) \mathbf{F}^{T}, \\
\mathbf{T}_{M} & =\mathbf{E} \otimes \mathbf{D}-\frac{\epsilon_{0}}{2} \mathbf{E} \cdot \mathbf{E} \mathbf{I} .
\end{aligned}
$$

The sum of $\boldsymbol{\sigma}$ and $\mathbf{T}_{M}$ is symmetric, though individually they may not be; and this is consistent with frame indifference in a polarizable continua. ${ }^{15,17}$

Eq. 18, 19, 20, 21, together with continuity equation 5 and 6 , are equations concerning the diffusions of $N_{d 0}$ and $\rho_{c 0}$. Since the diffusion of electrons is much faster than that of ions and vacancies, and we are primarily concerned with the latter, we assume that $\rho_{c 0}$ is in steady state in the time scale appropriate for $N_{d 0}$ 's diffusion. Therefore, we may regard $\rho_{c 0}$ as a known function of the electric potential $\phi$ and the donor density $N_{d 0}$ at any point and substitute this in Maxwell's equation 8. Therefore, the equations we need to solve are (see ${ }^{15}$ for detail):

$$
\begin{aligned}
& \frac{\partial N_{d 0}}{\partial t}-\nabla \cdot\left(\beta N_{d 0} \nabla\left(\frac{\partial W_{0}}{\partial N_{d 0}}+e z \phi\right)\right)=0 \quad \text { in } \Omega, \\
& \nabla \cdot\left(-\epsilon_{0} \nabla \phi+\mathbf{p} \chi(\mathbf{y}(\Omega))\right)=\rho\left(\phi, N_{d}\right) \quad \text { in } \mathbb{R}^{3}
\end{aligned}
$$


where $\beta=k_{1} / N_{d 0}$ is the defect's mobility. The assertion $\rho=\rho\left(\phi, N_{d}\right)$ may be regarded as an additional constitutive equation. Section 4 , we take it to be the charge density in a typical semiconductor in thermal equilibrium state is ${ }^{18}$ :

$$
\begin{aligned}
\rho\left(\phi, N_{d}, N_{a}\right)= & -e N_{c} F_{\frac{1}{2}}\left(\frac{E_{f m}-E_{c}+e \phi}{K_{b} T}\right)+e N_{v} F_{\frac{1}{2}}\left(\frac{E_{v}-e \phi-E_{f m}}{K_{b} T}\right) \\
& +z e N_{d}(x)\left(1-\frac{1}{1+\frac{1}{2} \exp \left(\frac{E_{d}-e \phi-E_{f m}}{K_{b} T}\right)}\right)-z^{\prime} e N_{a}(x)\left(1-\frac{1}{1+\frac{1}{2} \exp \left(\frac{E_{f m}+e \phi-E_{a}}{K_{b} T}\right)}\right) .
\end{aligned}
$$

where $N_{c}$ and $N_{v}$ are the effective density of states in the conduction band and in the valence band respectively; $E_{c}$ is the energy at the bottom of the conduction band, $E_{v}$ the energy on the top of the valence band, $E_{d}, E_{a}$ the donor and acceptor level respectively; $K_{b}$ is Boltzmann constant, $T$ the absolute temperature; $F_{\frac{1}{2}}$ is the Dirac-Fermi integral, and $E_{f m}$ is the Fermi level of the electrodes. Note that this expression allows for both donors with density $N_{d}$ and acceptors with density $N_{a}$.

In summary, the governing equations for a diffusible, deformable, ferroelectric semiconducting solids under external fields are:

$$
\begin{aligned}
\nabla_{\mathbf{x}}\left(\frac{\partial W_{0}}{\partial \nabla_{\mathbf{x}} \mathbf{p}_{0}}\right)-\frac{\partial W}{\partial \mathbf{p}_{0}}-\mathbf{F}^{-T} \nabla_{\mathbf{x}} \phi & =\mu \dot{\mathbf{p}}_{0} & & \text { in } \quad \Omega, \\
\frac{\partial W_{0}}{\partial \nabla_{\mathbf{x}} \mathbf{p}_{0}} \hat{\mathbf{m}} & =0 & & \text { on } \partial \Omega, \\
\left(\boldsymbol{\sigma}_{i j}+\mathbf{T}_{M i j}\right)_{, j} & =0 & & \text { in } \quad \mathbf{y}(\Omega), \\
\boldsymbol{\sigma} \hat{\mathbf{n}}-\llbracket \mathbf{T}_{M} \hat{\mathbf{n}} \rrbracket-\mathbf{t} \chi\left(\partial \mathbf{y}\left(\Omega_{s}\right)\right) & =0 & & \text { on } \partial \mathbf{y}(\Omega), \\
\frac{\partial N_{d 0}}{\partial t}-\nabla \cdot\left(\beta N_{d 0} \nabla\left(\frac{\partial W_{0}}{\partial N_{d 0}}+e z \phi\right)\right) & =0 & & \text { in } \Omega, \\
\nabla \cdot\left(-\epsilon_{0} \nabla \phi+\mathbf{p} \chi(\mathbf{y}(\Omega))\right) & =\rho\left(\phi, N_{d}\right) & & \text { in } \mathbb{R}^{3} .
\end{aligned}
$$

\section{DOMAIN WALLS IN PERFECT CRYSTALS}

We first consider perovskite ferroelectrics without defects in the form a $\mathrm{Pt} / \mathrm{BaTiO}_{3} / \mathrm{Pt}$ structure with shorted electrodes. $\mathrm{BaTiO}_{3}$ is nonpolar cubic above the Curie temperature but tetragonal distorted and polarized at room temperature. It is well known that for cubic paraelectric to tetragonal ferroelectric phase transition, only $180^{\circ}$ and $90^{\circ}$ twin boundaries can satisfy the mechanical and electrical compatibility conditions simultaneously. ${ }^{19}$ Here, we use finite element method to study the detailed structure of these two types of domain walls; we are specially interested in the strain, stress, and electric field near the domain walls. The advantage of using finite element method is that the computed variable is the displacement. Most simulations in the literature use finite difference with strain as their primary variable and this requires higher regularity. The disadvantages of using displacement as primary variables is the difficulty of applying periodic boundary conditions. For simplicity, we only consider small deformation here; therefore we will not differentiate current configuration from the reference configuration. This is a reasonable choice for $\mathrm{BaTiO}_{3}$ where the $c / a$ ratio for the tetragonal phase is 1.01. But note that it would be questionable for $\mathrm{PbTiO}_{3}$ where the $c / a$ ratio is about $1.06 .{ }^{19}$ We will also ignore the Maxwell stress here*. Under these assumptions, our system equations become:

$$
\begin{aligned}
& \mu \dot{\mathbf{p}}-\nabla\left(\frac{\partial W}{\partial \nabla \mathbf{p}}\right)+\frac{\partial W}{\partial \mathbf{p}}+\nabla \phi=0 \quad \text { in } \quad \Omega, \\
& \frac{\partial W}{\partial \nabla \mathbf{p}} \hat{\mathbf{n}}=0 \quad \text { on } \quad \partial \Omega, \\
& \boldsymbol{\sigma}_{i j, j}=0 \quad \text { in } \Omega, \\
& \boldsymbol{\sigma} \hat{\mathbf{n}}-\mathbf{t} \chi\left(\partial \Omega_{s}\right)=0 \quad \text { on } \quad \partial \Omega, \\
& \nabla \cdot\left(-\epsilon_{0} \nabla \phi+\mathbf{p} \chi(\Omega)\right)=0 \quad \text { in } \mathbb{R}^{3} .
\end{aligned}
$$

\footnotetext{
${ }^{*}$ It is reasonable according to our estimation. ${ }^{15}$
} 
We choose the stored energy function $W$ to be the Landau-Ginzburg-Devonshire energy with slight modification $^{20}$ :

$$
\begin{aligned}
W(\nabla \mathbf{p}, \mathbf{p}, \varepsilon) & =\frac{a_{0}}{2}\left(p_{x, x}^{2}+p_{x, y}^{2}+p_{y, x}^{2}+p_{y, y}^{2}\right) \\
& +\frac{a_{1}}{2}\left(p_{x}^{2}+p_{y}^{2}\right)+\frac{a_{2}}{4}\left(p_{x}^{4}+p_{y}^{4}\right)+\frac{a_{3}}{2} p_{x}^{2} p_{y}^{2}+\frac{a_{4}}{6}\left(p_{x}^{6}+p_{y}^{6}\right)+\frac{a_{5}}{4}\left(p_{x}^{4} p_{y}^{4}\right) \\
& -\frac{b_{1}}{2}\left(\varepsilon_{x x} p_{x}^{2}+\varepsilon_{y y} p_{y}^{2}\right)-\frac{b_{2}}{2}\left(\varepsilon_{x x} p_{y}^{2}+\varepsilon_{y y} p_{x}^{2}\right)-b_{3} \varepsilon_{x y} p_{x} p_{y} \\
& +\frac{c_{1}}{2}\left(\varepsilon_{x x}^{2}+\varepsilon_{y y}^{2}\right)+c_{2} \varepsilon_{x x} \varepsilon_{y y}+\frac{c_{3}}{2} \varepsilon_{x y}^{2}
\end{aligned}
$$

We nondimensionalize ${ }^{20}$ as: $x_{i}{ }^{\prime}=x \sqrt{c_{0} / a_{0}} / p_{0}, p_{i}{ }^{\prime}=p_{i} / p_{0}, \mu^{\prime}=1, t^{\prime}=t c_{0} / \mu p_{0}{ }^{2}, \epsilon_{0}{ }^{\prime}=\epsilon_{0} c_{0} / p_{0}{ }^{2}, \phi^{\prime}=\phi / \sqrt{a_{0} c_{0}}$, $a_{0}{ }^{\prime}=1, a_{1}{ }^{\prime}=a_{1} p_{0}{ }^{2} / c_{0}, a_{2}{ }^{\prime}=a_{2} p_{0}{ }^{4} / c_{0}, a_{3}{ }^{\prime}=a_{3} p_{0}{ }^{4} / c_{0}, a_{4}{ }^{\prime}=a_{4} p_{0}{ }^{6} / c_{0}, a_{5}{ }^{\prime}=a_{5} p_{0}{ }^{8} / c_{0}, b_{j}{ }^{\prime}=b_{j} p_{0}{ }^{2} / c_{0}$ and $c_{j}{ }^{\prime}=c_{j} / c_{0}$, where $i=1,2$ and $j=1,2,3$. For $\mathrm{BaTiO}_{3}$, the spontaneous polarization in room temperature is $0.26 \mathrm{C} / \mathrm{m}^{2}$. Therefore, we choose $p_{0}=0.26 \mathrm{C} / \mathrm{m}^{2}$ such that the normalized spontaneous polarization 1 . The material constants we choose $\operatorname{are}^{20}: c_{0}=1 \mathrm{GPa}, \epsilon_{0}{ }^{\prime}=0.131, c_{1}{ }^{\prime}=185, c_{2}{ }^{\prime}=111, c_{3}{ }^{\prime}=54, b_{1}{ }^{\prime}=1.4282$, $b_{2}{ }^{\prime}=-0.185, b_{3}{ }^{\prime}=0.5886, a_{1}{ }^{\prime}=-0.007, a_{2}{ }^{\prime}=-0.009, a_{3}{ }^{\prime}=0.003, a_{4}{ }^{\prime}=0.0261, a_{5}{ }^{\prime}=5$.

As we can see that the $\nabla \mathbf{p}$ term in the energy functional penalizes rapid changes of polarizations, therefore decides the thickness of domain walls: the thickness of domain walls is actually proportional to $\sqrt{a_{0} / a_{1}}$. The advantage of this normalization is that we do not need to worry about choosing proper $a_{0}$ in order to generate realistic domain wall thickness; the specimen size is adjusted according to the domain wall thickness.

Since we will use finite element method to simulate the domain wall structure, it's advantageous to rewrite $W$ as:

$$
\begin{aligned}
W(\nabla \mathbf{p}, \mathbf{p}, \varepsilon) & =\frac{a_{0}}{2}\left(p_{x, x}^{2}+p_{x, y}^{2}+p_{y, x}^{2}+p_{y, y}^{2}\right) \\
& +\frac{a_{1}}{2}\left(p_{x}^{2}+p_{y}^{2}\right)+\left(\frac{a_{2}}{4}-d\right)\left(p_{x}^{4}+p_{y}^{4}\right)+\left(\frac{a_{3}}{2}-f\right) p_{x}^{2} p_{y}^{2}+\frac{a_{4}}{6}\left(p_{x}^{6}+p_{y}^{6}\right)+\frac{a_{5}}{4}\left(p_{x}^{4} p_{y}^{4}\right) \\
& +\frac{1}{2}\left(\varepsilon-\varepsilon_{s}\right) \cdot \mathbf{C}\left(\varepsilon-\varepsilon_{s}\right),
\end{aligned}
$$

where $\mathbf{C}$ is the stiffness matrix,

$$
\mathbf{C}=\left(\begin{array}{ccc}
c_{1} & c_{2} & 0 \\
c_{2} & c_{1} & 0 \\
0 & 0 & c_{3}
\end{array}\right)
$$

$\varepsilon_{s}$ the eigenstrain caused by spontaneous polarization:

$$
\varepsilon_{s}=\left(a p_{x}{ }^{2}-b p_{y}{ }^{2} \quad b p_{x}{ }^{2}-a p_{y}{ }^{2} \quad c p_{x} p_{y}\right)^{T}
$$

and

$$
\begin{aligned}
a & =\frac{b_{1} c_{1}-b_{2} c_{2}}{2\left(c_{1}^{2}-c_{2}^{2}\right)}, \\
b & =\frac{b_{2} c_{1}-b_{1} c_{2}}{2\left(c_{1}^{2}-c_{2}^{2}\right)} \\
c & =\frac{b_{3}}{c_{3}} \\
d & =\frac{-2 b_{1} b_{2} c_{2}+\left(b_{1}^{2}+b_{2}^{2}\right) c_{1}}{8\left(c_{1}^{2}-c_{2}^{2}\right)}, \\
f & =\frac{2 b_{1} b_{2} c_{1}-\left(b_{1}{ }^{2}+b_{2}{ }^{2}\right) c_{2}}{4\left(c_{1}{ }^{2}-c_{2}{ }^{2}\right)}-\frac{b_{3}^{2}}{2 c_{3}} .
\end{aligned}
$$



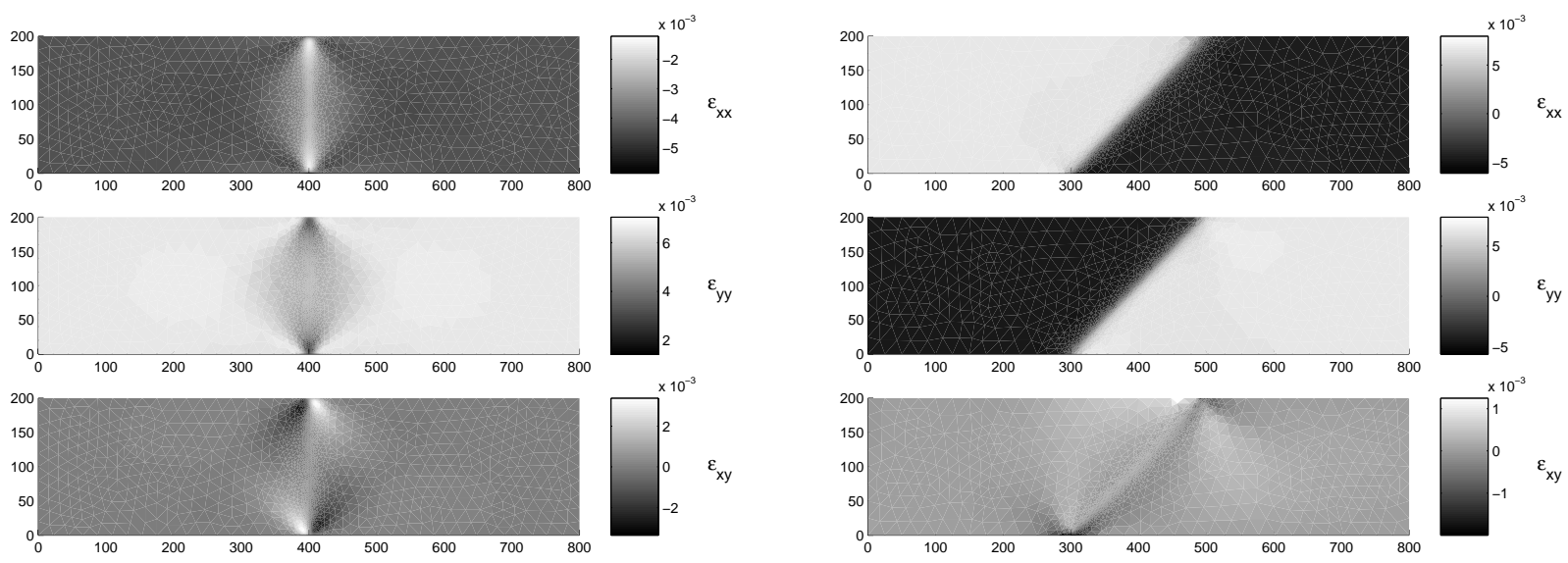

Figure 2. Strain profile near $180^{\circ}$ (left) and $90^{\circ}$ (right) domain wall.
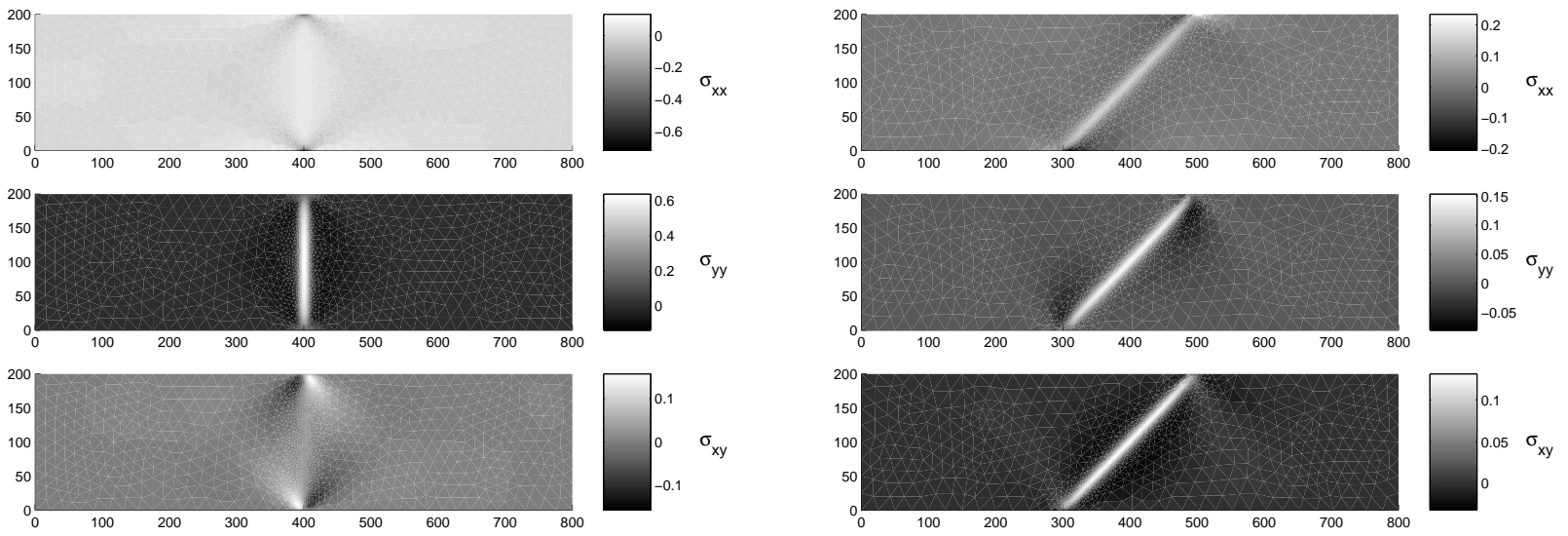

Figure 3. Stress profile near $180^{\circ}$ (left) and $90^{\circ}$ (right) domain wall(GPa).

We compute on a $800 \times 200$ rectangular domain here. The boundary conditions we choose are: upper or lower boundary shorted, no flux at left and right side; no rotation/reflection allowed, no displacement at left lower corner point, no $x$ displacement along left side, stress free on other sides.

Figure 2 and 3 are the strain and stress field near $180^{\circ}$ and $90^{\circ}$ domain walls. It is easy to see that the strain/stress are raised near the domain walls, and concentrations at the intersections of domain walls with electrodes. These strain/stress concentration sites may likely serve as starting points for microcracking or domain wall pinning. It is also interesting to notice that there is not much difference in terms of magnitude of stress concentration between $180^{\circ}$ and $90^{\circ}$ domain walls. This is a little surprising, since the conventional wisdom states that $90^{\circ}$ domain walls undergo much more distortion than $180^{\circ}$ domain walls.

Figure 4 is the potential profile across $180^{\circ}$ and $90^{\circ}$ domain walls. We notice that the potential across $90^{\circ}$ domain wall is one order larger than the potential across $180^{\circ}$ domain walls. This tells us that $90^{\circ}$ domain walls indeed have much larger electrical mismatch than $180^{\circ}$ domain walls. It is exactly this electrical mismatch which makes a $90^{\circ}$ domain walls a plausible site for electronic charge trapping as we will see in Section 4 .

\section{OXYGEN VACANCIES AS DEFECTS}

We now turn our attention to $\mathrm{BaTiO}_{3}$ with defects. We still consider the $\mathrm{Pt} / \mathrm{BaTiO} / \mathrm{Pt}$ structure, but with oxygen vacancies in $\mathrm{BaTiO}_{3}$. For simplicity, we look at a single snap-shot in time when the oxygen vacancy 
concentration is uniform, and also ignore the effects of elasticity. Therefore, the equations reduce to:

$$
\begin{aligned}
\mu \dot{\mathbf{p}}-\nabla\left(\frac{\partial W}{\partial \nabla \mathbf{p}}\right)+\frac{\partial W}{\partial \mathbf{p}}+\nabla \phi & =0 \quad \text { in } \quad \Omega, \\
\frac{\partial W}{\partial \nabla \mathbf{p}} \hat{\mathbf{n}}=0 & \text { on } \quad \partial \Omega, \\
\nabla \cdot\left(-\epsilon_{0} \nabla \phi+\mathbf{p} \chi(\Omega)\right)=\rho(\phi) & \text { in } \quad \mathbb{R}^{3}
\end{aligned}
$$

with $\rho(\phi)$ defined in Eq. 26.

\subsection{Interaction of Defects with Domain Walls}

We first investigate the electrostatic consequences of the interaction between the oxygen vacancies and domain walls. Still using finite element method, we compute a $400 \times 200$ rectangular domain, with a $180^{\circ}$ or a $90^{\circ}$ domain wall in the middle. We assume the density of oxygen vacancies $N_{d}=1.0 \times 10^{24} \mathrm{~m}^{-3}$. We choose $a_{0}=1.0 \times 10^{-7} \mathrm{Vm}^{3} \mathrm{C}^{-1}$ here, the film thickness is therefore about $530 \mathrm{~nm}$. The constants of band structures
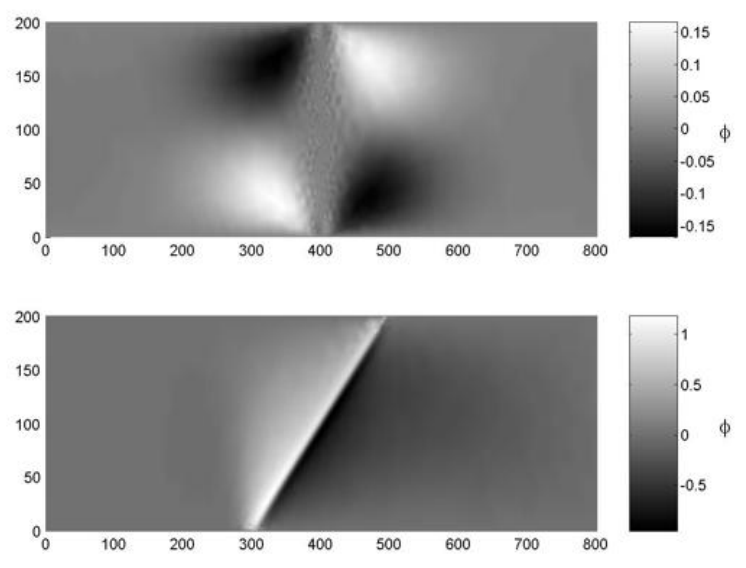

Figure 4. Electric potential near $180^{\circ}$ (upper) and $90^{\circ}$ (lower) domain walls. The real value of $\phi$ depends on $a_{0}$, the number $(\mathrm{V})$ shown here is by choosing $a_{0}=1.0 \times 10^{-7} \mathrm{Vm}^{3} \mathrm{C}^{-1}$.
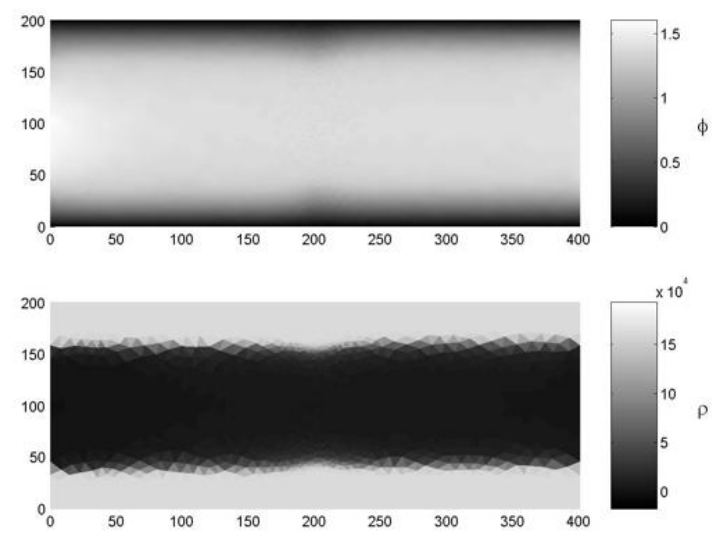
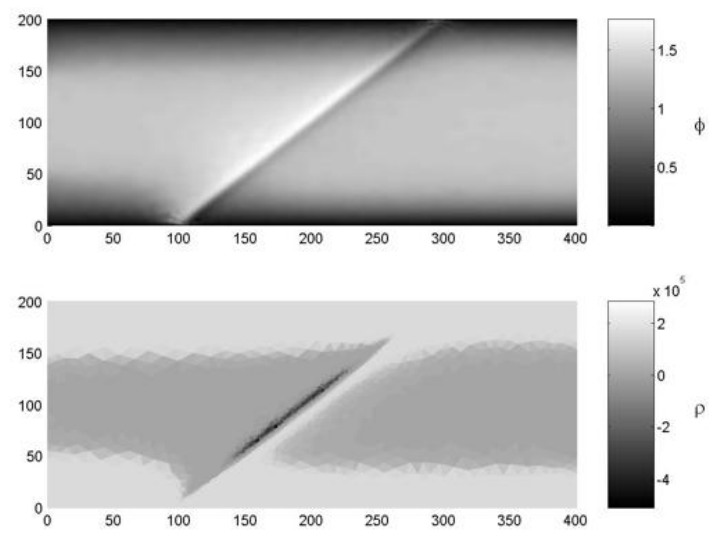

Figure 5. Electric potential(V) and charge densities $\left(\mathrm{C} \mathrm{m}^{-3}\right)$ near $180^{\circ}$ (left) and $90^{\circ}$ (right) domain walls 

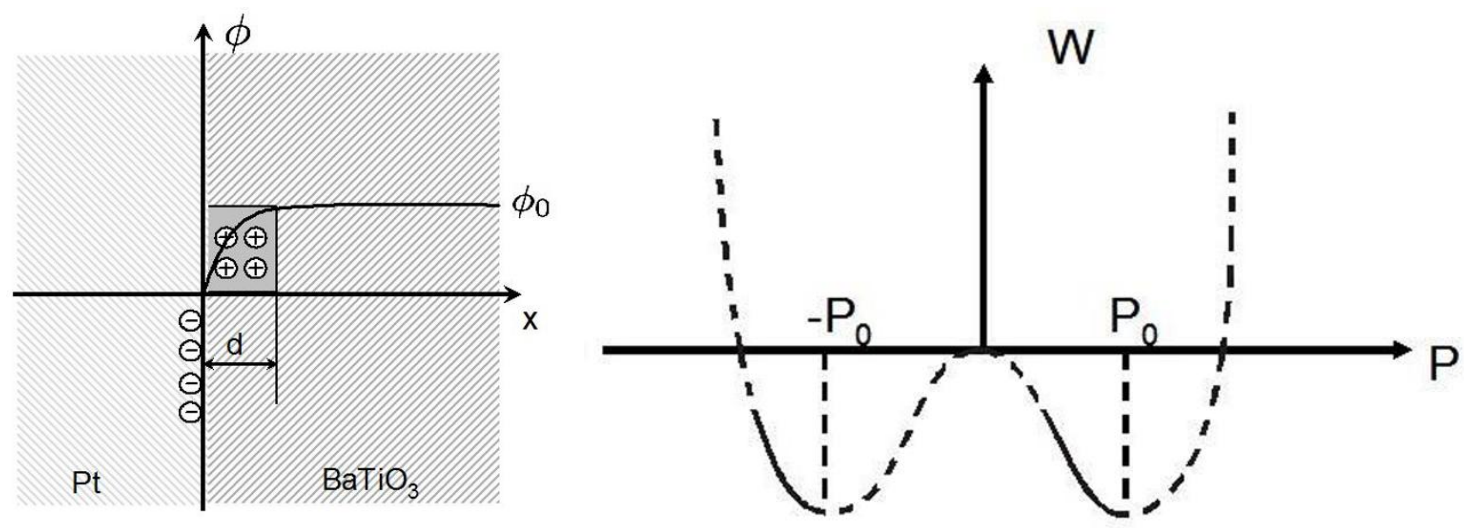

Figure 6. Left: Potential profile across $\mathrm{Pt} / \mathrm{n}$-type $\mathrm{BaTiO}_{3}$ interface. Right: Double well energy for ferroelectric crystal: the reversal of the solid line is the potential profile in the depletion layer on the left.

of $\mathrm{Pt}$ and $\mathrm{BaTiO}_{3}$ are chosen to be ${ }^{9}: E_{f m}=-5.3 \mathrm{eV}$ (corresponding to the work function of Pt of $5.3 \mathrm{eV}$ ), $E_{c}=-3.6 \mathrm{eV}, E_{d}=-4.0 \mathrm{eV}, E_{a}=-6.2 \mathrm{eV}, E_{v}=-6.6 \mathrm{eV}$.

The computational result is shown in Figure 5. This figure shows only the $\mathrm{BaTiO}_{3}$ and the $\mathrm{Pt}$ electrodes are on the top and bottom surface. The first difference we immediately notice from the case with no defects (Figure 4) is the sharp rise in electrostatic potential from the electrodes to the interior of the film by as much as $1.5 \mathrm{~V}$. This is caused by the the diffusion of electrons from $\mathrm{BaTiO}_{3}$ into Pt giving rise to "depletion layers" of approximate thickness of 100nm. These depletion layers also generate the Schottky barriers across the interfaces. The Schottky barriers are important in preventing dielectric breakdown by resisting the injection of charges into the ferroelectric. The large electric field in the depletion layer however provides a driving force for the field-driven diffusion of oxygen vacancies. This in turn lowers the Schottky barrier and promotes dielectric breakdown.

In the case of the $180^{\circ}$ domain walls (left of Figure 5), the depletion layer overwhelms any contribution from the domain wall and we barely notice the domain wall in either the distribution of potential or charges. Thus we conclude that $180^{\circ}$ domain walls has very little interaction with oxygen vacancies and effect on their diffusion.

In contrast, there is a significant interaction between $90^{\circ}$ domain walls and oxygen vacancies as shown on the right side of Figure 5. We see the depletion layers as before, but we also see that very large amounts of negative charges are accumulated along the domain wall. In other words, we have electrons injected from electrodes and trapped at the domain wall at equilibrium. The reason for this can be understood by going back to the the electric potential in Figure 4 without defects and noting the large electric field at the domain wall. This drives the injection of charges into the domain wall. Despite this, an electric field remains at the domain wall as shown in Figure 5 and this can in turn can force the diffusion of oxygen vacancies and lead to pinning of domain walls.

In summary, we find that the field caused by the depletion layers near the electrodes can promote oxygen vacancy diffusion and lowering of the Schottky barrier. We also find that 90 degree domain walls can contribute to dielectric breakdown by promoting the injection of charges and to fatigue by pinning of domain walls.

\subsection{Band Bending and Depletion Layers}

We now derive approximate analytic description of the profile of the electric field and the width of the depletion layer. In light of the band structures of $\mathrm{Pt}$ and $\mathrm{BaTiO}_{3}$ (see Section 4.1), we expect the electrons on $\mathrm{BaTiO}_{3}$ 's side to flow to Pt side which has a lower energy. Along with the Schottky barrier generated across the interface, a positive charged layer near the interface on $\mathrm{BaTiO}_{3}$ sides is also formed; and the potential profile shall be as shown schematically in Figure 6.

We approximate $\rho(\phi)$ given by Eq. 26 as follows: when $\phi$ is small, say smaller than $0.3 \mathrm{~V}, \rho \approx z e N_{d}$; when $\phi$ 
is large, say larger than $0.3 \mathrm{~V}$, but smaller than $\left(E_{c}-E_{f m}\right) / e$, the charge density is:

$$
\rho \approx-e N_{c} \exp \left(\frac{E_{f m}-E_{c}+e \phi}{K_{b} T}\right)+z e N_{d}(x)\left(1-\frac{1}{1+\frac{1}{2} \exp \left(\frac{E_{d}-e \phi-E_{f m}}{K_{b} T}\right)}\right)
$$

We need charge neutrality deep in the ferroelectric crystal, i.e, $\rho$ in Eq. 50 should be zero with $\phi=\phi_{0}$. We obtain,

$$
\phi_{0} \approx \frac{1}{2 e}\left(E_{c}+E_{d}-2 E_{f m}\right)
$$

For $\mathrm{BaTiO}_{3}, \phi_{0} \approx 1.5 \mathrm{~V}$, which agrees very well with our computation results for $180^{\circ}$ domain walls.

We now turn to the detailed potential profile cross the depletion layer. We need to solve following equation:

$$
\begin{aligned}
& -\epsilon_{0} \phi_{, x x}+p_{, x}=\rho \\
& \frac{\partial W(p)}{\partial p}+\phi_{, x}=0
\end{aligned}
$$

with

$$
\rho=\left\{\begin{array}{lll}
e z N_{d} & \text { if } & 0<x<d \\
0 & \text { if } & x>d
\end{array}\right.
$$

For a dielectric semiconductor where $W(p)=p^{2} /\left(2 \chi \epsilon_{0}\right)$, i.e. $p=\epsilon_{0} \chi E$, the above equation is easy to solve and the answer is well known. The width of depletion layer in this case shall be

$$
d=\sqrt{2 \epsilon_{r} \epsilon_{0} \phi_{0} / \rho} .
$$

where $\epsilon_{r}=1+\chi$ is the relative dielectric constant.

For a ferroelectric semiconductor, however, things are not so straightforward. Since $\epsilon_{0}$ is small, we follow Shenoy and Bhattacharya ${ }^{9}$ neglect that term in Eq. 52 and solve:

$$
\begin{aligned}
p_{, x} & =\rho \\
\frac{\partial W(p)}{\partial p}+\phi_{, x} & =0
\end{aligned}
$$

with $W(p)$ the usual polynomial with double-well shape as in Figure 6 . It is easy to see that $p=\rho x+k_{1}$, where $k_{1}$ is some constant. And

$$
\phi(x)=\int-\frac{d W}{d p}\left(\rho x+k_{1}\right) d x+k_{2}=\int-\frac{d W}{d p} \frac{d x}{d p} d p+k_{2}=-\frac{1}{\rho} W\left(\rho x+k_{1}\right)+k_{2}
$$

where $k_{2}$ is some constant.

Assuming that far from the interface, we have $p=p_{0}$ (or $p=-p_{0}$ depending on the polarization direction), we obtain

$$
p=p_{0}+\rho(x-d) \quad \text { when } \quad 0<x<d .
$$

Therefore, we have:

$$
\begin{gathered}
\phi(0)=-\frac{1}{\rho} W\left(p_{0}-\rho d\right)+k_{2}=0, \\
\phi(d)=-\frac{1}{\rho} W\left(p_{0}\right)+k_{2}=\phi_{0},
\end{gathered}
$$

which leads to:

$$
\phi_{0} \rho=W\left(p_{0}-\rho d\right)-W\left(p_{0}\right) .
$$


We perform a Taylor expansion of the right-hand-side and note that for a ferroelectric crystal with $\mathrm{W}(\mathrm{p})$ as described by Figure $6, \frac{\partial W}{\partial p}\left(p_{0}\right)=0$. We finally obtain:

$$
d=\sqrt{\frac{2 \phi_{0}}{\rho}\left(\frac{\partial^{2} W\left(p_{0}\right)}{\partial p^{2}}\right)^{-1}}
$$

This is similar to the result for a dielectric semiconductor if we set:

$$
\epsilon_{r}=\left(\frac{\partial^{2} W\left(p_{0}\right)}{\partial p^{2}} \epsilon_{0}\right)^{-1}
$$

With the material constants we have chosen for $\mathrm{BaTiO}_{3}, \epsilon_{r} \approx 113$. If $N_{d}=1.0 \times 10^{24} \mathrm{~m}^{-3}$, then the depletion layer is about $100 \mathrm{~nm}$ thick, which is consistent with our computation results in Section 4.1. Finally notice that as the oxygen vacancy concentration $N_{d}$ increases, so does $\rho$ resulting in a reduction of $d$ and the Schottky barrier.

\section{CONCLUSION}

We have developed a continuum theory for a diffusible, deformable, ferroelectric semiconducting solids under external fields. We also investigated the domain wall structures both in perfect crystals and in defected crystals. Based on our simulation, we believe ionic defects (oxygen vacancies) and electronic charges play important roles in ferroelectric fatigue. To be specific, that the existence of oxygen vacancies in the perovskite ferroelectric thin film, especially, their accumulation near the electrodes lowers Schottky barrier and thus accelerates electronic charge injections. Further $90^{\circ}$ domain walls are perfect trapping sites for injected electronic charges. The trapped electronic or ionic charges may lead to domain wall pinnings, or eventually lead to fatigue.

\section{ACKNOWLEDGMENTS}

We thank Vivek Shenoy and Wei Zhang for useful discussions. We are also glad to acknowledge the financial support of the US Army Research Office through the MURI grant No. DAAD 19-01-1-0517.

\section{REFERENCES}

1. J. Scott, Ferroelectric Memories, Springer, 2000.

2. D. Damjanovic, "Ferroelectric, dielectric and piezoelectric properties of ferroelectric thin films and ceramics," Rep. Prog. Phys 61, pp. 1267-1324, 1998.

3. A. Tagantsev, I. Stolichnov, E. Colla, and N. Setter, "Polarization fatigue in ferroelectric films: Basic experimental findings, phenomenological scenarios, and microscopic features," J. Appl. Phys. 90(3), pp. 1387$1402,2001$.

4. T. Shaw, S. Trolier-Mckinstry, and P. McIntyre, "The properties of ferroelectric films at small dimensions," Annu. Rev. Mater. Sci. 30, pp. 263-298, 2000.

5. W. Warren, D. Dimos, and et al, "Electronic domain pinning in $\mathrm{Pb}(\mathrm{Zr}, \mathrm{Ti}) \mathrm{O}_{3}$ thin films and its role in fatigue," Appl. Phys. Lett. 65(8), pp. 1018-1020, 1994.

6. W. Warren, D. Dimos, and et al, "Polarization suprresion in $\mathrm{Pb}(\mathrm{Zr}, \mathrm{Ti}) \mathrm{O}_{3}$ thin filmes," J. Appl. Phys. 77(12), pp. 6695-6702, 1995.

7. B. Gütter, U. Bismayer, P. Groves, and E. Salje, "Fatigue mechanism in thin film PZT memory materials.," Semicond. Sci. Technol. 10, pp. 245-248, 1995.

8. B. Nagaraj, S. Aggarwal, and R. Ramesh, "Influence of contact electrodes on leakage characteristics in ferroelectric thin films," J. Appl. Phys. 90(1), pp. 375-382, 2001.

9. V. Shenoy and K. Bhattacharya, "Effect of doping on polarization profile and switching in ferroelectric thin films," To be submitted.

10. R. Ramesh and et al, "Fatigue and retention in ferroelectric $\mathrm{Y}-\mathrm{Ba}-\mathrm{Cu}-\mathrm{O} / \mathrm{Pb}-\mathrm{Zr}-\mathrm{Ti}-\mathrm{O} / \mathrm{Y}-\mathrm{Ba}-\mathrm{Cu}-\mathrm{O}$ heterostructures," Appl. Phys. Lett. 61(13), pp. 1537-1539, 1992. 
11. C. DeAraujo, J. Cuchiaro, and et al, "Fatigue-free ferroelectric capacitors with platinum electrodes," Nature 374(6523), pp. 627-629, 1995.

12. J. Scott, C. Araujo, B. Melnick, L. McMillan, and R. Zuleeg, "Quantititative measurement of space-charge effects in lead zirconate-titanate memories," J. Appl. Phys. 70(1), pp. 382-388, 1991.

13. L. Sanchez and et al, "Processing and characterization of sol-gel derived very thin film ferroelectric capacitors,"

14. J. Scott, K. Watanabe, A. Hartmann, and R. Lamb, "Device models for PZT/Pt, BST/Pt, SBT/Pt, and SBT/Bi ferroelectric memories," Ferroelectrics 225, pp. 83-90, 1999.

15. Y. Xiao and K. Bhattacharya, "A continuum model of diffusible, deformable, ferroelectric semiconducting solids under external fields," In preparation.

16. R. Coleman and W. Noll, "The thermodynamics of elastic materials with heat conduction and viscosity," Arch. Rational Mech. Anal 13(3), pp. 167-178, 1963.

17. R. Toupin, "The elastic dielectric," J. Rational Mech. Anal. 5, pp. 849-915, 1956.

18. S. Sze, Physics of Semiconductor Devices, Wiley, 1981.

19. Y. Shu and K. Bhattacharya, "Domain patterns and macroscopic behavior of ferroelectric materials," Phylosophical Magazine B 81(12), pp. 2021-2054, 2001.

20. W. Zhang and K. Bhattacharya, "Modeling large strain eletrostriction of ferroelectrics under combined electromechanical loads," in Proceedings of the SPIE Conference on Smart Structure and Materials 2003, D. Lagoudas, ed., 5053, 2003. 